\section{Copacabana Method for Spreading $E$. coli and Yeast Colonies}

BioTechniques 30:738-742 (April 2001)

Molecular biology techniques require the isolation of individual clones for analysis after bacterial or yeast transformation. For E. coli and Saccharomyces cerevisiae, the dispersion of colonies on plates has traditionally involved the use of a glass spreader-a bent glass rod that could be repeatedly flame sterilized and used to spread a small aliquot of microorganisms from the center of the plate to the periphery to cover the entire plate $(1,2)$. For many investigators, learning to disperse bacteria on solid media is one of their first exposures to molecular biology techniques.

We describe an alternative method for using sterile glass balls for the dispersion of bacteria and yeast on solid media that requires less technical skill on the part of the operator and avoids smearing colonies or damaging the agar. It also eliminates the need for breakable glass spreaders and open containers of ethanol required for repeated flaming.

Glass balls (4 mm) obtained from Thomas Scientific (Swedesboro, NJ, USA) were autoclaved in 100-mL milk dilution bottles. One Shot ${ }^{\mathrm{TM}}$ Top10 Competent cells and the pBADThio TOPO $^{\mathrm{MM}}$ TA Cloning Kit were obtained from Invitrogen (Carlsbad, CA, USA). A PCR product was generated in our laboratory, ligated into the vector, transformed into the bacteria per the manufacturer's directions, and spread on LB media containing $50 \mu \mathrm{g} / \mathrm{mL}$ carbenicillin. A TRPl-containing yeast expression vector was transformed into the strain Y499 and plated onto tryptophan-deficient complete minimal yeast media using standard methods (3).

For $E$. coli plating, three to five sterile glass balls were shaken onto a 100$\mathrm{mm} \mathrm{LB} /$ carbenicillin plate after the plate had been warmed in an oven at approximately $40^{\circ} \mathrm{C}$ with the lid vented to remove condensation. The glass balls were dispensed by flaming the cap and neck of the milk dilution bottle and gently shaking them out of the bottle a few centimeters above the media, three or four per $100-\mathrm{mm}$ plate. E. coli transformation mixture $(50-150 \mu \mathrm{L})$ was aliquoted onto the center of the media, and the lid was placed back onto the plate. Then, we agitated the plate with a random shaking motion so that the glass balls would roll over the entire surface of the plate. Once the liquid is absorbed and the surface of the media is dry, this process is finished (usually $30-60 \mathrm{~s}$ if the plate has been sufficiently pre-warmed). The dry plate is inverted, the beads discarded, and we incubate the media at $37^{\circ} \mathrm{C}$ overnight. Similar volumes and numbers of beads are used for yeast media for selection by complementation of auxotrophic markers, such as uracil, tryptophan, or leucine-deficient complete minimal media for yeast one- and two-hybrid screens. For either organism, if the plate size is larger, we scale the number of glass balls and volume of transformation mixture proportionately. For example, for a $150-\mathrm{mm}$ plate, we routinely plate $350 \mu \mathrm{L}$ transformation mixture and use 8-12 glass balls (Table 1).

A laboratory strain of $E$. coli was transformed with the product of a cloning reaction and plated onto the selective media using the Copacabana method. The glass balls are shown in Figure 1A on the surface of a LB/carbenicillin plate before plating. A plate suitable for this purpose allows the balls to move freely with the lid in place; plates with less than $5 \mathrm{~mm}$ between the surface of the media and the inner surface of the lid cannot be used. The 100-mm plates we use in our labo-

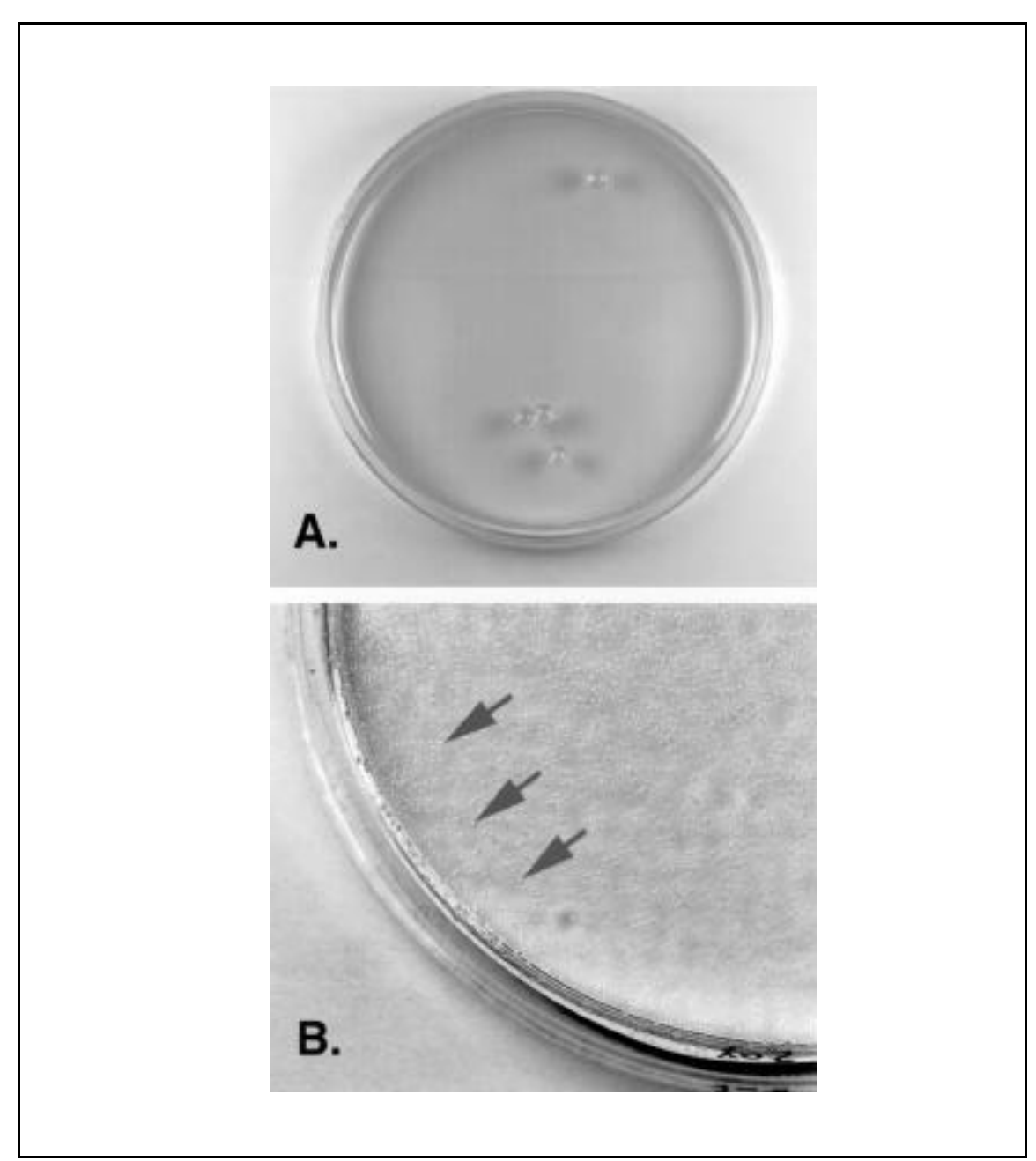

Figure 1. The Copacabana spreading method leads to good dispersion of $E$. coli colonies. (A) Four glass beads are shown on a $100-\mathrm{mm}$ bacterial plate, ready for the introduction of the transformation mixture. (B) The results of the Copacabana method are shown after an overnight incubation with many isolated colonies available for clonal analysis. Black arrows indicate a few representative colonies. 


\section{Benchmarks}

Table 1. The Copacabana Method for Spreading E. coli and Yeast Transformations

1. Vent the plates in a warm oven or incubator until all visible condensation is gone. (We use a glassware oven set at approximately $40^{\circ} \mathrm{C}$ while the transformation mixture is shaking).

2. Close and move the dried plates onto the bench top. Add the requisite number of sterile beads and transformation mixture based on the size of the plate.

\section{Plate Size}

100-mm round

$150-\mathrm{mm}$ round

88-mm square

\section{No. of Beads}

$3-5$
$8-12$
$3-5$

Transformation Mixture ( $\mu \mathrm{L})$

$$
\begin{array}{r}
50-150 \\
300-400
\end{array}
$$$$
50-150
$$

3. Agitate the plate to uniformly spread the glass balls and the transformation mixture over the surface of the plate.

4. Once the liquid has been absorbed and the plate has dried, decant the beads into the laboratory waste, invert the plate, and incubate at the desired temperature.

Notes: For plate sizes not listed, experiment with the number of beads and amount of transformation mixture, based on the surface area of the plate. For volumes less than those listed, add an appropriate liquid medium for the plate to the desired volume (e.g., LB or SOC for E. coli transformations). This can also be used to add additional antibiotics or chemicals to previously poured plates.

ratory (Fisher Scientific, Pittsburgh, PA, USA) have approximately $13 \mathrm{~mm}$ between the base and the lid before the medium is poured. In Figure $1 \mathrm{~B}, 50 \mu \mathrm{L}$ transformation mixture were plated onto a similar $100-\mathrm{mm}$ plate, and the uniformity of spreading over the plate can be clearly seen. Figure 2 shows $S$.

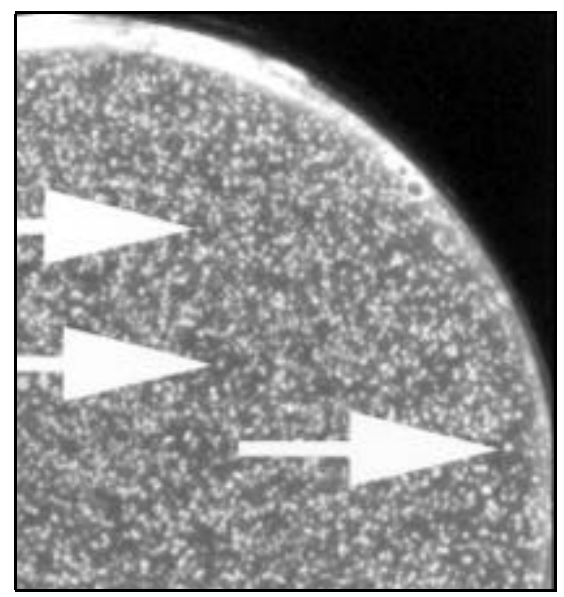

Figure 2. S. cerevesiae transformants are well dispersed and unsmeared with the use of the Copacabana method. Competent YPH499 $S$. cerevisiae was transformed with a constitutive TRPI plasmid and plated on tryptophan-deficient complete minimal media. White arrows indicate a few representative yeast colonies. No colonies were seen on a transformation plate without plasmid DNA (not shown). cerevisiae transformed with a TRP1 plasmid plated onto Trp media. Although at a lower density than the bacterial transformations, the evenness of dispersion is clearly seen.

After using this method to spread over a thousand bacterial and yeast plates, we have eliminated the need for glass spreaders and open beakers of ethanol. This technique is easily taught to laboratory novices. Three investigators who were inexperienced with the glass bead method but expert with the traditional method were asked to plate three transformation mixtures using both methods. Although trends existed for faster plating time and more distinct colonies per plate with the Copacabana method, this did not reach statistical significance $(P<0.05$; not shown). Parenthetically, all three of these investigators now prefer the Copacabana method.

A single investigator can easily spread ten $150-\mathrm{mm}$ plates at a time, agitating five in each hand for library transformations, and then spreading the next ten, etc. If a single transformation mixture is diluted with SOC media to the appropriate volume, a single investigator can quickly spread two to 10 million cfu of a library transformation. With a relatively small colony size $E$. coli bacterium, such as DH10B, TOP10, or XL-
1 strains, densities of greater than or equal to 50000 transformants on a 150$\mathrm{mm}$ plate are not impossible. It is useful for round and squared plates and could conceivably be automated for highthroughput applications. Unlike the glass spreader method, the operator does not have to develop the feel for ineffective pressure versus pressure that damages the media.

There are some tricks to using glass balls for this process. We limit the num ber of glass balls from three to five on a $100-\mathrm{mm}$ plate and are careful to use a random motion when spreading. This results in the characteristic "clacking" sound as each portion of the plate is covered. The "Roller Derby" method of swirling the beads around the periphery of the plate should be avoided-the goal is to repeatedly cover the surface of the plate with the moist glass beads until the medium is dry. The warm, dried plates are elastic, and shaking them from too great a height can result in bouncing the balls onto the bench top or floor. Upon completion, we decant the glass balls into our laboratory waste.

At the time of this publication, the cost of four glass balls is less than $\$ 0.03$ per $100-\mathrm{mm}$ plate. Inexpensive 4-mm glass balls can be obtained from most suppliers and will last most laboratories several years (VWR Scientific, Bridgeport, NJ, USA; PGC Scientifics, Gaithersburg, MD, USA; Thomas Scientific, Swedesboro, NJ, USA). In theory, the balls could be reused if they are washed and re-sterilized.

In conclusion, the Copacabana method is a useful, easily mastered alternative for plating bacteria and yeast on solid media and avoids smearing colonies or damaging the agar. It eliminates the need for breakable glass spreaders and open containers of ethanol for flaming.

\section{REFERENCES}

1.Lech, K. and R. Brent. 1994. Escherichia coli: media preparation and bacteriological tools, p. 1.1.1-1.2.2. In F. Ausubel, R. Brent, R. Kingston, D. Moore, J. Seidman, J. Smith, and K. Struhl (Eds.), Current Protocols in Molecular Biology. John Wiley and Sons, New York.

2.Sambrook, J., E.F. Fritsch, and T. Maniatis. 1989. Preparation and transformation of com petent E. coli, p. 1.74-1.84. In Molecular 
Cloning: A Laboratory Manual. CSH Laboratory Press, Cold Spring Harbor, New York.

3.Treco, D. and V. Lundblad. 1997. Basic techniques of yeast genetics, p. 13.1.1-13.1.7. In F. Ausubel, R. Brent, R. Kingston, D. Moore, J. Seidman, J. Smith, and K. Struhl (Eds.), Current Protocols in Molecular Biology. John Wiley and Sons, New York.

This work was supported in part by $\mathrm{Na}$ tional Institutes of Health grant no. DK02501 and by the American Digestive Health Foundation. This work is dedicated to the memory of Daniel Nathans and his trainees. Special thanks to Tim Schaefer for helpful discussions and to Phil Hieter for his gift of yeast strain YPH499. Address correspondence to Dr. Mark T. Worthington, MR-4 Building, Room 1036, 1 Lane Road, UVAHSC, Charlottesville, VA 22908, USA.e-mail:mtw3p@virginia.edu

Received 2 August 2000; accepted December 2000.

\section{Mark T. Worthington, Roger Qi Luo, and Jared Pelo University of Virginia Health Sciences Center Charlottesville, VA, USA}

\section{Incorporation of Sodium Sulfite into Extraction Protocol Minimizes Degradation of Acacia DNA}

\author{
BioTechniques 30:742-748 (April 2001)
}

The isolation of high-quality DNA from plants, especially woody plants, is often difficult. Although there are many different methods for DNA extraction in use, it is not unusual to find plant species for which commonly used extraction methods do not produce goodquality DNA. The main reason for the inability to obtain high-quality genom ic DNA from Australian native plants is the high levels of cytosolic compounds such as polysaccharides, polyphenolics, and tannins.

A reliable DNA extraction procedure for eucalypts (5) was developed by modification of a method of Wagner et al. (9) and has been successfully used to extract high-quality DNA from other Australian native species such as Lam bertia orbifolia (4) and Macrozamia riedlei (6). The procedure is based on a crude organelle isolation from which the DNA is extracted using a cetyltrimethylammonium bromide (CTAB) method. Modifications to this protocol have been used to obtain high-quality DNA from other species. The addition of high levels of salt to the extraction protocol, followed by a caylase treatment, resulted in the removal of polysaccharide contamination from Acacia mangium DNA (2). In Melaleuca alternifolia, degradation of DNA during restriction enzyme digestion was prevented by the incorporation of a differential solvent precipitation into the extraction procedure (3).

The use of the modified Wagner method (5) to isolate DNA from a num ber of Acacia species (A. acuminata, A. anfractuosa, A. lobulata, A. sciophanes, and $A$. verricula) resulted in degradation of DNA immediately after extraction, as determined by agarose gel electrophoresis (Figure 1). Several modifications to the protocol were tested, including the ones noted above, but the best results were obtained by the addition of $0.1 \mathrm{M}$ sodium sulfite to the two buffers used in the extraction procedure (the initial extraction buffer and the buffer used to resuspend the organelle pellet). No other modifications to the modified Wagner extraction procedure were necessary when sodium sulfite was used, and no other modifications were successful when sodium sulfite was not used. The addition of sodium sulfite resulted in extraction of highquality DNA that was not degraded, while the standard protocol produced DNA that was degraded (Figure 1). The DNA extracted with sodium sulfite was suitable for use in restriction fragment length polymorphism (RFLP) analyses. Figure 2 shows the effect on RFLP analysis of the DNA extracted with and without the addition of sodium sulfite to the buffers. The high molecular weight DNA extracted with the addition of sodium sulfite to the extraction buffers gives a clean hybridization profile, while the degraded DNA obtained from the standard method gives a poor hybridization profile with smeared lanes and a weak hybridization signal.

The direct action of sodium sulfite in the extraction procedure is unclear. Sodium sulfite is a reducing agent for polyphenol oxidase, and its presence will prevent the production of polyphenolic compounds. It seems likely that there is a nuclease present in the extraction solution that becomes bound with a phenolic compound and is not removed by the organic extraction. Prevention of phenolic formation by the addition of sodium sulfite would leave the nuclease free to be removed during the organic extraction. Alternatively, there may be a compound in the phenolic pathway that has nuclease properties.

Other studies of Acacia species using RFLP techniques have also encountered problems with the quality of DNA. Polysaccharide contamination was problematic in A. mangium(2) and a number of other Acacia species (1), and another study required purification of DNA through cesium chloride gradi-

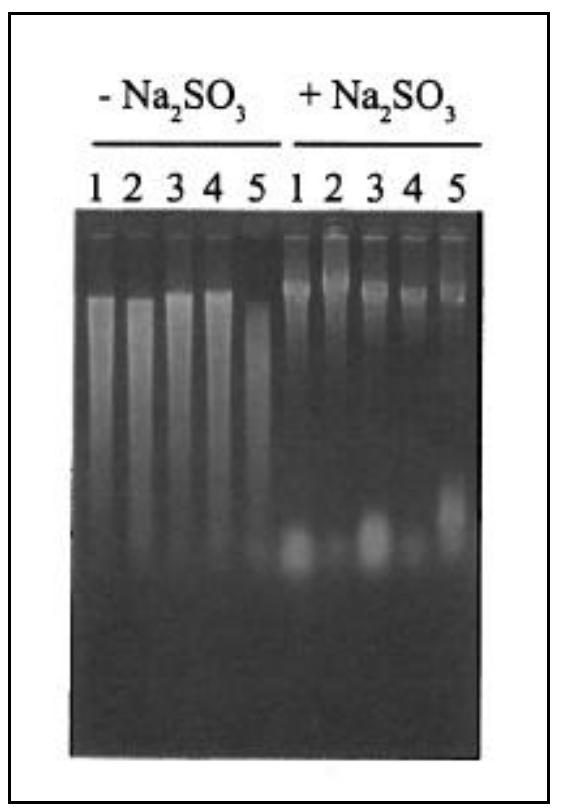

Figure 1. A. verricula DNA extracted with and without addition of sodium sulfite to the buffers in the modified Wagner extraction method. Lanes 1-5, DNA extracted from the Merredin population without using sodium sulfite in the extraction buffers. Lanes 6-10, DNA extracted from the Merredin population with the addition of sodium sulfite to the extraction buffers. 